\title{
Three-dimensional Reconstruction of Carotid Arteries using Computed Tomography Angiography
}

\author{
Vassiliki I. Kigka ${ }^{1}$, Savvas Kyriakidis ${ }^{1,2}$, Antonis Sakellarios ${ }^{1,2}$, Vassiliki Potsika ${ }^{1}$, \\ Vasilis Tsakanikas ${ }^{1}$, Dimitra Loggitsi ${ }^{3}$, Lampros K. Michalis ${ }^{4}$, Dimitrios I. Foti- \\ adis $1,2, *$ \\ ${ }^{1}$ Unit of Medical Technology and Intelligent Information Systems, Department of Materials \\ Science and Engineering, University of Ioannina, GR 45110, Ioannina, Greece. \\ ${ }^{2}$ Institute of Molecular Biology and Biotechnology, Dept. of Biomedical Research Institute - \\ FORTH, University Campus of Ioannina, GR 45110, Ioannina, Greece. \\ ${ }^{3}$ CT/MRI Department, Hygeia \& Mitera Hospitals, Athens, Greece \\ ${ }^{4}$ Department of Cardiology, Medical School, University of Ioannina, GR 45110, Ioannina, \\ Greece.
}

\begin{abstract}
Carotid artery disease is considered as the pathological disease of carotid arteries and is considered as a principal cause of stroke. Therefore, early diagnosis of carotid artery disease is of high clinical importance. This study aims to present an overall methodology for the accurate identification of the inner wall, outer wall and the atherosclerotic plaques (calcified and non-calcified) of the carotid arteries. The proposed methodology is based on a level set based approach, which is fully adapted in each computed tomography acquisition protocol. Briefly, the methodology includes the following steps: (i) the estimation of intensity membership functions for the inner wall, the outer wall and $\mathrm{CP}$, (ii) the carotid artery centerline extraction, (iii) the inner wall, outer wall and calcified plaques segmentation, (iv) the noncalcified plaques segmentation and finally (v) the 3D models construction. The segmentation accuracy of the proposed methodology has been validated against manual expert's annotations in 4 patients, and more specifically in 300 computed tomography angiography slices for the inner wall segmentation and in 30 slices as far as the atherosclerotic plaques is concerned. The utilized evaluation metrics were the Dice coefficient and the Hausdorff Distance and our very first results are promising for the accurate and automated segmentation of carotid arteries.
\end{abstract}

Keywords: carotid artery disease, three-dimensional reconstruction, computed tomography coronary angiography, level set segmentation

\section{Introduction}

Carotid artery disease is considered as a major cause of stroke and its underlying cause is usually atherosclerosis. Atherosclerosis is a disease in which atherosclerotic plaque, consisting of fat, cholesterol, calcium and other substances, builds up and narrows the arteries. Carotid artery disease early diagnosis and carotid artery stenosis 
(CAS) detection is of high importance, and therefore different carotid artery disease imaging modalities have been developed, including the duplex ultrasound (DUS), the computed tomography angiography (CTA) and the magnetic resonance angiography (MRA). DUS is basically recommended for an initial CAS diagnostic examination, whereas CTA and MRA are non-invasive imaging modalities, recommended for further diagnostic examination of the CAS, the intracranial cerebral circulation, the aortic arch condition and generally of the progression of carotid artery disease [1].

Computed Tomography Angiography (CTA) is a non-invasive imaging modality utilized for the assessment of the carotid stenosis. The main advantage of the CTA imaging is the ability to image the artery bifurcations, since it is able to image from the aortic arch to the brain parenchyma [2], whereas the high radiation dosage for the need of intravenous contrast and the high intensity artifacts consist of the acknowledged drawbacks of CTA imaging modality.

Different studies have been conducted for the identification and characterization of carotid atherosclerotic plaques, which can be distinguished into intraplaque haemorrhage, fibrous cap, lipid-rich core and calcification. CTA imaging modality has been considered to be the best imaging modality for the detection of calcifications in the carotid plaque. Manual segmentation of carotid arteries is a time consuming and tedious procedure and is highly depended on the expert observer's ability to detect artery lumen borders. Thus, there is a strong need for the development of computer-aided segmentation procedure both for the lumen detection and the atherosclerotic plaque detection and characterization.

In the literature, different methodologies have been proposed for the analysis of the carotid arteries, based on CTA imaging modality. More specifically, Sanderse et al. [3] presented an automatic initialization algorithm to detect the carotid arteries providing a fully automated approach for vessel centerline detection and segmentation. van Velsen et al. [4] introduced a study that is based on the detection of lumen-like structures by analyzing the density, edge and ridge knowledge. Manniesing and Niessen (2007) [5] presented an automatic method that segments the internal carotid arteries across the difficult locations of the skull base in CTA. In another study proposed by Cuisenaire [6], a fully automated vessel extraction and segmentation tool specifically used for head and neck region was developed. Vukadinovic et al. [7] presented a levelset based and GentleBoost classification based approach for the segmentation of the carotid artery outer vessel wall and the atherosclerotic plaque components, whereas Freiman et al. [8] proposed a practical methodology for the modeling of the total aortic arch and of the entire carotid vasculature, starting with an automated region of interest (ROI) identification which is followed by the implementation of an automatic watershed-based segmentation of the aorta. In a more recent study, Tang et al. [9] proposed a semi-automatic carotid lumen segmentation method and carotid stenosis quantification using a level-set based approach, which was initiated with a centerline obtained from user defined seed points, whereas in 2018 Bozkurt et al. [10] provided a novel method for carotid artery lumen segmentation on CTA images by using automatic vessel segmentation with inverse approach.

The aim of this study is to present a fully-automated methodology for the detailed identification of the carotid artery anatomy and the identification of the carotid artery atherosclerotic plaques and their distribution over the 3D space. 


\section{Methodology}

In this section, the overall 3D reconstruction methodology will be presented. An extended algorithm for the 3D reconstruction of carotid arteries atherosclerotic plaques using CTA imaging modality was developed and calibrated based on the CTA images quality, the CTA scanners and the acquisition dose protocols.

The 3D reconstruction algorithm aims basically to segment the inner wall, the outer wall of the carotid arteries and to identify the calcified plaques (CP) and the noncalcified plaques (NCP) into the region of interest (ROI). Basically the utilized segmentation methodology is a 3D level set methodology as far as the inner wall, outer wall and CP segmentation is concerned, whereas the NCP pixels are identified based on dynamic thresholding technique. The main concept of the proposed methodology is that it is adapted in all acquisition dose protocols and CTA images derived by different CTA scanners. Overall, the proposed methodology includes different steps:

i.the estimation of intensity membership functions for the inner wall, the outer wall and $\mathrm{CP}$,

ii.carotid artery centerline extraction,

iii.inner wall segmentation

iv.outer wall segmentation

v.CP segmentation

vi.NCP segmentation

vii.3D models construction.

Briefly three different sigmoidal membership functions are estimated for the three components (the inner wall, the outer wall and the $\mathrm{CP}$ ). These membership functions aim to correspond its pixel to an inner wall, outer wall or CP pixel and are estimated with two different ways. The overall concept of these membership functions use is to adapt the intensity range for each component on the value of mean lumen intensity pixels. These membership functions are defined either based on fixed thresholds derived by the literature, or based on threshold defined by the user after a calibration procedure. In the second step, a minimum cost path based approach proposed by Metzt et al.[11] for the carotid artery centerline extraction is applied. In the third, fourth and fifth step a 3D level set approach using prior shapes is applied for the segmentation of the inner wall, the outer wall and the CP, whereas in the sixth step a dynamic threshold technique is implemented for the NCP segmentation. Finally, a triangulation methodology approach is applied for the 3D models construction.

The proposed methodology is an extension of an already existing methodology for the $3 \mathrm{D}$ reconstruction of coronary arteries $[12,13]$ and is calibrated for the carotid arteries. In addition to this, the overall methodology has been incorporated into a software tool, which is easily used and requires by the user the minimum interaction (only the starting and the ending point) of each vessel. In addition to this, the proposed methodology is able to provide not only carotid segment, but also carotid bifurcations. Bifurcation artery regions are high risk regions for atherosclerotic plaque growth. 
In Figure 1 below, we demonstrate (a) the 3D reconstruction of a carotid artery bifurcation, (b) the 2D CTCA slice without segmentation and (c) the 2D segmentation of the inner wall and outer wall.

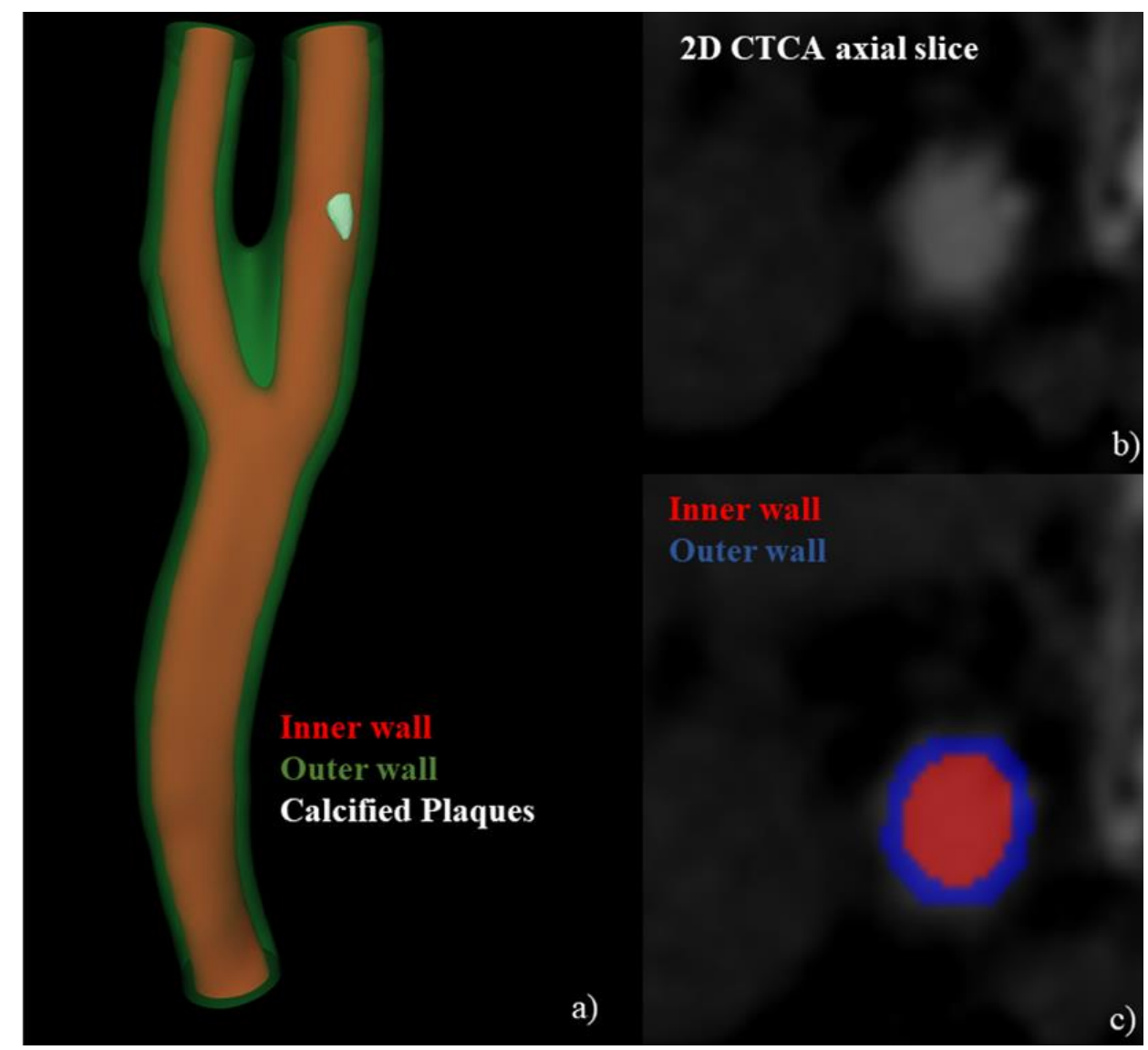

Fig. 1. :(a) 3D reconstruction of a carotid artery bifurcation, (b) the 2D CTCA slice without segmentation and (c) the 2D segmentation of the inner wall and outer wall.

\section{Validation}

The aim of the validation process is to assess the accuracy and the quality of the proposed methodology. In this study, our purpose is to validate our methodology, using as gold standard a medical expert's annotations. It is known that the lack of expertannotated datasets remains one of the main challenges in medical image processing [20]. Generating high-quality expert-derived annotations in CTA images is time-consuming and requires a specialized in CTA imaging field medical expert. 
Reconstruction of carotid arteries, both for the left common artery (LCA) and the right common artery (RCA), was successfully obtained in 4 patients. Our very first results indicated a value of DICE coefficient is $0.76 \pm 0.07$, whereas the Hausdorff distance is $1.51 \pm 0.36$, as far as the inner wall detection is concerned. The respective evaluation metrics for the identification of $\mathrm{CP}$ is $0.71 \pm 0.07$ and $1.63 \pm 0.22$, whereas the Dice coefficient and the Hausdorff distance for the NCP are $0.7 \pm 0.04$ and $1.63 \pm 0.18$, respectively. 


\section{Discussion}

Different studies have been proposed in the literature for the carotid arteries CTA imaged analysis. Most of them are dedicated on the entire carotid vessel (outer wall) detection $[3,5-7,10]$ and do not identify the inner wall (lumen) of the carotid arteries. On the other hand, Freiman et al. [8] in a study proposed in 2012 presented a graph-cut based segmentation algorithm for the accurate detection of carotid artery lumen (inner wall) and achieves an overall DICE similarity of $84.5 \% \pm 3.3 \%$. Nevertheless, this study is validated against experimental existing studies [14] and not directly compared to experts' manual annotations, which is considered as the gold standard. Moreover, another carotid artery lumen segmentation methodology has been presented by Hemmati et al. [15] and achieves an overall DICE coefficient of 0.85. However, this study is not capable of identifying the vessel centerline of small branches and it is necessary to remove small branches in centerline extraction stage. Additionally, the methodology fails to identify the vessel centerline in the regions where the vessel is occluded. Other existing studies, such as the study proposed by Guha et al. [16] and dos Santos et al.[17] have not been validated quantitively.

Our proposed study for the segmentation of the entire carotid artery vasculature and the atherosclerotic plaques presented a complete methodology for the segmentation of the two anatomical layers of the carotid arteries, both the inner wall and the outer wall. The detection of both the inner and the outer wall of the carotid artery constitutes the novel aspect of the proposed methodology, since both layers have a clinical significance. More specifically, the degree of stenosis, calculated by the segmentation of the inner wall, is considered as one of the most significant geometric features of the carotid artery and its value contributes to the clinical decision and the patient management. Additionally, the quantification of the overall plaque burden region, computed by the detection of both the inner wall and outer wall, is also a significant measurement as far as the carotid artery disease prediction and its progression is concerned. Another advantage of our presented methodology it its incorporation in a user friendly software tool, which provides the 2D segmentation in each $2 \mathrm{D} \mathrm{CT}$ axial slice, the $3 \mathrm{D}$ rendering of both the inner wall and outer wall of the carotid arteries and the geometrical based metrics.

\section{Conclusion}

CTA imaging modality is considered as a promising noninvasive technique for the identification of the carotid arteries and for the diagnostic examination of carotid artery disease. This integrated methodology provides accurate 3D models of carotid arteries, also utilized for the blood flow modeling and plaque growth modeling prediction. Our next steps are to validate the proposed methodology utilizing more patients and to implement computational modeling for the carotid artery disease stratification. 


\section{References}

[1] A. Saxena, E. Y. K. Ng, and S. T. Lim, "Imaging modalities to diagnose carotid artery stenosis: progress and prospect," Biomedical engineering online, vol. 18, p. 66, 2019.

[2] A. Huibers, G. De Borst, S. Wan, F. Kennedy, A. Giannopoulos, F. Moll, et al., "Noninvasive carotid artery imaging to identify the vulnerable plaque: current status and future goals," European Journal of Vascular and Endovascular Surgery, vol. 50, pp. 563-572, 2015.

[3] M. Sanderse, H. A. Marquering, E. A. Hendriks, A. van der Lugt, and J. H. C. Reiber, "Automatic Initialization Algorithm for Carotid Artery Segmentation in CTA Images," in Medical Image Computing and Computer-Assisted Intervention - MICCAI 2005, Berlin, Heidelberg, 2005, pp. 846-853.

[4] E. F. S. van Velsen, W. J. Niessen, T. T. de Weert, C. de Monyé, A. van der Lugt, E. Meijering, et al., "Evaluation of an improved technique for lumen path definition and lumen segmentation of atherosclerotic vessels in CT angiography," European Radiology, vol. 17, pp. 1738-1745, July 012007.

[5] R. Manniesing and W. J. Niessen, "Automatic segmentation of the internal carotid arteries through the skull base," in Medical Imaging 2007: Image Processing, 2007, p. 65120I.

[6] O. Cuisenaire, S. Virmani, M. E. Olszewski, and R. Ardon, "Fully automated segmentation of carotid and vertebral arteries from contrast enhanced CTA," in Medical Imaging 2008: Image Processing, 2008, p. 69143R.

[7] D. Vukadinovic, T. van Walsum, R. Manniesing, S. Rozie, A. van der Lugt, and W. J. Niessen, "Region based level set segmentation of the outer wall of the carotid bifurcation in CTA," in Medical Imaging 2011: Image Processing, 2011, p. 79623P.

[8] M. Freiman, L. Joskowicz, N. Broide, M. Natanzon, E. Nammer, O. Shilon, et al., "Carotid vasculature modeling from patient $\mathrm{CT}$ angiography studies for interventional procedures simulation," International Journal of Computer Assisted Radiology and Surgery, vol. 7, pp. 799-812, 2012/09/01 2012.

[9] H. Tang, T. van Walsum, R. Hameeteman, R. Shahzad, L. J. van Vliet, and W. J. Niessen, "Lumen segmentation and stenosis quantification of atherosclerotic carotid arteries in CTA utilizing a centerline intensity prior," Medical physics, vol. 40, 2013.

[10] F. Bozkurt, C. Köse, and A. Sar1, "An inverse approach for automatic segmentation of carotid and vertebral arteries in CTA," Expert Systems with Applications, vol. 93, pp. 358-375, 2018.

[11] C. Metz, M. Schaap, A. Weustink, N. Mollet, T. van Walsum, and W. Niessen, "Coronary centerline extraction from CT coronary angiography images using a minimum cost path approach," Medical physics, vol. 36, pp. 5568-5579, 2009.

[12] V. I. Kigka, G. Rigas, A. Sakellarios, P. Siogkas, I. O. Andrikos, T. P. Exarchos, et al., "3D reconstruction of coronary arteries and atherosclerotic plaques based on computed tomography angiography images," Biomedical Signal Processing and Control, vol. 40, pp. 286-294, 2018.

[13] V. I. Kigka, A. Sakellarios, G. Rigas, P. Tsobou, I. O. Andrikos, L. K. Michalis, et al., "A Three-Dimensional Quantification of Calcified and Non-calcified Plaque Based on Computed Tomography Coronary Angiography Images: Comparison with Virtual 
Histology Intravascular Ultrasound," in World Congress on Medical Physics and Biomedical Engineering 2018, 2019, pp. 207-211.

[14] K. Hameeteman, M. A. Zuluaga, M. Freiman, L. Joskowicz, O. Cuisenaire, L. F. Valencia, et al., "Evaluation framework for carotid bifurcation lumen segmentation and stenosis grading," Medical image analysis, vol. 15, pp. 477-488, 2011.

[15] H. Hemmati, A. Kamli-Asl, A. Talebpour, and S. Shirani, "Semi-automatic 3D segmentation of carotid lumen in contrast-enhanced computed tomography angiography images," Physica Medica, vol. 31, pp. 1098-1104, 2015.

[16] I. Guha, N. Das, P. Rakshit, M. Nasipuri, P. K. Saha, and S. Basu, "A semiautomatic approach for segmentation of carotid vasculature from patients' CTA images," Innovations in Systems and Software Engineering, vol. 13, pp. 243-250, 2017.

[17] F. L. C. dos Santos, A. Joutsen, M. Terada, J. Salenius, and H. Eskola, "A semiautomatic segmentation method for the structural analysis of carotid atherosclerotic plaques by computed tomography angiography," Journal of atherosclerosis and thrombosis, vol. 21, pp. 930-940, 2014. 